\title{
Referencing an approved protocol in an amendment
}

\begin{abstract}
Dr. Erica Waite had two IACUC-approved protocols. Her first protocol, identified by the IACUC as M22-16, included the use of mice in type 2 diabetes studies. That protocol and the NIH grant that supported her research specifically included the use of glucose tolerance tests (GTTs) and insulin tolerance tests (ITTs) on the mice. Waite also had a second NIH grant and a second IACUC protocol (M33-16). M33-16 also included the use of mice in type 2 diabetes studies, but the needs of this research only required performing GTTs on the animals. Both her grants and IACUC protocols adequately described the methodologies for performing the required tolerance tests.
\end{abstract}

About six months into study M33-16, Waite's research revealed insulin-related findings that were totally unexpected but quite interesting. She submitted an amendment to her IACUC protocol in which she requested to perform ITTs on a cohort of the mice. Waite wrote in the amendment that she would be using the exact same methodology and equipment that was already approved for the animals in M22-16. However, the IACUC office staff informed her that she could not simply reference the methodology used in M22-16; rather, she would have to rewrite the entire methodology into the amendment request for M33-16.
That made no sense to Waite. She asked the IACUC office why in the past she was able to indicate on a protocol form that the school's transgenic mouse core would construct genetically modified mice for her, yet she did not have to include all the details of the surgery that occurred in the transgenic core? She wanted to know why she was allowed to reference the transgenic core, which had an IACUC-approved protocol, but not her own M22-16 protocol.

Did the IACUC office give Waite the correct regulatory information? What is the reasoning behind your opinion and how would you approach this question?

\section{RESPONSE}

\section{Methods are too important to gloss over}

\author{
Jessica K. Lang, BSc, BUS, rLATG, CM \& \\ Laike Stewart, DVM, DACLAM
}

We believe that the IACUC gave Waite accurate information. The experimental design of a project is often the most important part of a protocol for how a PI plans to answer the questions for which the described research is being undertaken ${ }^{1,2}$. Each IACUC-approved protocol and its associated amendments should be stand-alone documents. Simply referring to her other approved protocol does not satisfy that requirement.

The word 'core' implies that the pertinent research is run by the animal facility and therefore might have more regulatory oversight or overlap with the IACUC. Additionally, the IACUC is probably much more familiar with that protocol since there are multiple users using the core facility. Furthermore, the creation of transgenic mice is likely a procedure that most IACUC members have familiarity with, unlike the ITTs. Therefore, it would be redundant to require that each PI using the core recopy the entire construct process into their individual protocols. We would approach Waite's question by explaining to her that it is easier to have access to a complete centralized protocol than to need to refer to another document.

1. Petrie, W.K. \& Wallace, S.L. The Care and Feeding of an IACUC: The Organization and Management of an Institutional Animal Care and Use Committee 2nd edn. (CRC Press, Boca Rotan, FL, 2015).

2. Institute for Laboratory Animal Research. Guide for the Care and Use of Laboratory Animals 8th edn. (National Academies Press, Washington, DC, 2011).

Lang is a Senior Research Specialist and Gnotobiotic Facility Manager at Harvard T.H. Chan School of Public Health, Boston, MA. Stewart is the Associate Director and Associate Professor at the Division of Veterinary Resources, University of Miami Miller School of Medicine, Miami, FL.

\section{RESPONSE}

\section{All references are not created equal}

\section{Jamie Lewis, BA, CPIA, RLATG \& \\ Judy Barnett, BS, CMAR, RLATG}

We assume that Waite's research is PHSfunded; as such, statements in the PHS Policy on Humane Care and Use of Laboratory Animals regarding protocol content are crucial to answering whether the IACUC staff gave her correct information. The policy states that "the IACUC shall confirm that the research project ... is consistent with the Guide"1.

The Guide for the Care and Use of Laboratory Animals describes protocol content as "a detailed description of the proposed use of laboratory animals. The following topics should be considered in the preparation of the protocol by the researcher and its review by the IACUC: a clear and concise sequential description of the procedures involving the use of animals that is easily understood by all members of the committee...impact of the proposed procedures on the animal's well-being.... appropriate sedation, analgesia and anesthesia" ${ }^{2}$.

Clearly the intent is for the protocol to describe what is being done to the animals and understand how pain and distress from the procedures will be mitigated. Does it need to be a stand-alone document to do this? The regulations do not cover whether methodology descriptions in protocols can reference other documents, but there is guidance on standard operating procedures (SOPs) from OLAW. PHS specifically allows IACUCapproved SOPs to be used for "routine 